\title{
Research on Correction Methods of Errors in Multi-beam Sounding Resulted from Water Flow
}

\author{
Xin Wang ${ }^{1}$ \\ ${ }^{1}$ Department of surveying and mapping, Shandong University of Science and Technology, Qingdao, Shandong, 266000, China
}

\begin{abstract}
The multi-beam sounding system combines multi-beam bathymetry and integrated navigation technology, which can obtain underwater topography data quickly and accurately, it has become the most important way of marine investigation. Therefore, it is urgent for improving the accuracy of sounding system in the detection industry. During the study of multi-beam error processing, the installation error coefficient of transducer installation will be corrected, but all the time, the transducer mounting bracket is regarded would be corrected. However, the transducer mounting bracket has been regarded as a rigid body in the correction for a long time. In this paper, the transducer bracket is regarded as a non-rigid body, and the reversible elastic deformation of transducer bracket occurs due to the impact of water flow. With the change of ship speed and water flow velocity, the degree of elastic deformation is also time-varying. The elastic deformation of bracket leads to the deflection of transducer shafting, which can not be corrected when correcting the installation deviation, so that leading to the influence of this error in final sounding results. This paper aims at the fact of deformation of multi-beam bracket caused by the water flow velocity, ship speed etc., leading to the deflection of transducer relative to the deviation of inertial navigation system shafting and resulting in attitude deviation which affect accuracy of result is researched.
\end{abstract}

\section{Introduction}

With the development of economy, the progress of science and technology as well as the increase of demands, the land resources seem to be increasingly insufficient, so the exploration and exploitation of resources have begun to go deep into the marine field. But all ocean-related activities require accurate submarine topographic data as support. Multi-beam sounding technology is a kind of fullcoverage submarine measuring technology, which has become the main method of submarine topographic measurement in large scale. However, under the impact of water flow, the multi-beam bracket may occur elastic deformation, thus causing deviation of the transducer shafting relative to the inertial navigation shafting, and the attitude deviation is generated by the inconsistency between measuring attitude of inertial navigation system

$$
\left\{\begin{array}{l}
\sin r^{\prime}=\sin \alpha \times \sin p+\cos \alpha \times \sin r \\
\sin p^{\prime}=\cos \alpha \times \sin p-\sin \alpha \times \sin r
\end{array}\right.
$$

In the formula, $r$ refers to the actual roll; $p$ refers to the actual pitch; the $\mathrm{r}^{\prime}$ is the measured roll; the $\mathrm{p}^{\prime}$ is the measured pitch.

It is easy to know from formula (1) that the attitude deviation is related to the real-time attitude and shafting deflection angle. and actual attitude of transducer, which further affects the accuracy of sounding results and the effect of swath join.

\section{Attitude deviation caused by water flow}

As shown in figure 1, it is assumed that the transducer and inertial navigation coordinate system are fully aligned with the carrier body coordinate system when installed, only the origin is different. During the measurement process, the bracket is deformed because of water flow impact and a deflection angle $\alpha$ of the transducer and the inertial navigation coordinate system around the $\mathrm{z}$ axis is produced. At this time, the relationship between the actual roll and pitch of transducer and the measured roll and pitch of inertial navigation system is shown as follows:

When there is still a roll deviation $\delta_{r}$ around the $\mathrm{Y}$ axis of the ship coordinate system after installing the deviation correction on transducer shafting and inertial navigation system shafting, on the assumption that there is only one layer of water depth, without considering the influence brought about by acoustic ray refraction, the approximate value of attitude deviation on water depth is 


$$
E_{\mathrm{V}}=Z\left[1-\frac{\cos \left(\theta+\delta_{r}\right)}{\cos (\theta)}\right]
$$

When there is still a pitch deviation $\delta_{p}$ around the X axis of the ship coordinate system after installing the error correction on transducer shafting and inertial navigation

$$
E_{\mathrm{V}}=Z\left(1-\cos \left(\delta_{P}\right)\right)
$$

system shafting, on the assumption that there is only one layer of water depth, without considering the influence brought about by acoustic ray refraction, the approximate value of attitude deviation on water depth is:

In formula (2) and formula (3), $E_{V}$ refers to the error of water depth; $\theta$ refers to the beam angle; $\delta_{r}$ refers to

the roll error; $\delta_{p}$ refers to the pitch error; $\mathrm{Z}$ refers to the measured water depth.

$\sim_{\mathrm{x}_{\mathrm{T}}} \mathrm{x}_{\mathrm{A}}$

Because this type of attitude deviation is caused by elastic deformation of bracket due to the water flow, both its symbol and size are related to the velocity of water flow and the output speed of ship. Different from the speed of ship relative to the ground, the output speed of ship refers to the speed of ship that is relative to the water body, which is only in relation to the engine power of ship and has nothing to do with the direction and size of water flow. For general multi-beam measurement, the velocity of water flow is not measured by Acoustics Doppler Current Profile (ADCP) as a supplementary, and the power of ship engine is changing all the time, that is, there is no direct

$$
\left\{\begin{array}{c}
\left|V_{\text {water }}\right| \times \sin \alpha=\left|V_{\text {ship }}\right| \times \sin \beta \\
\left|V_{\text {water }}\right| \times \cos \alpha+\left|V_{\text {ship }}\right| \times \cos \beta=|V|
\end{array}\right.
$$

In the formula, $V_{\text {water }}$ represents the velocity of water flow, $V_{\text {ship }}$ represents the output speed of ship, $\mathrm{V}$ is the final resultant velocity of the ship under measurement. According to the GNSS system, $\alpha$ refers to the sharp angle constructed by the direction of water flow and the direction of the ship, $\beta$ refers to the sharp angle constructed by the output speed of ship's propeller under measurement and the direction of ship, it can be calculated from the real-time course of ship and the azimuth of survey line that both $\alpha$ and $\beta$ are positive.

In most cases, hypothesis 1 and 2 are in line with the actual situation.

In the formula (4), water flow velocity $V_{\text {water }}$ and the output speed of ship can not be measured directly, the resultant velocity $\mathrm{V}$ of the ship can be calculated through ship's movement trajectory that is measured by GNSS system. $\beta$ represents the sharp angle constructed by the output speed of ship's propeller and the direction of the ship. There is no direct data on the direction about output measurement data for both water flow velocity and ship output speed. But because the ship's actual movement speed is the vector sum of ship's output speed and water flow, so they can be calculated according to the direction of the ship and the actual motion of the ship.

In order to facilitate analysis, the following hypotheses are made:

1. the direction and velocity of water flow are consistent in a short time;

2. the velocity of water flow, the output speed and the resultant velocity of ship conform to the parallelogram law, that is:

speed of the ship's propeller. However, in order to ensure good measurement results, the inertial navigation system shafting will maintain parallel relationship with the ship's shafting as far as possible during the process of installing the system, so the course data measured by the inertial navigation system are used to indicate the ship's orientation, in addition, the ship's moving direction is calculated through ship's movement trajectory that is measured by the GNSS positioning system, angle $\beta$ can be obtained therefrom.

As shown in Figure 2, the motion state of ship under measurement at a certain time is decomposed. Among them, point $A$ and point $B$ represent two points on the same survey line, decomposing the measured ship's state at point $\mathrm{A} ; \mathrm{H}$ indicates the course of measured ship at point A, which is provided by the inertial navigation system; $H_{\text {line }}$ represents the coordinate azimuth of survey line from point $\mathrm{A}$ to point $\mathrm{B}$, which can be calculated from their coordinates; $\gamma$ refers to the coordinate azimuth of 
water flow direction at point $\mathrm{A} ; V_{\text {water }}, V_{\text {ship }}$ and $\mathrm{V}$ represents the water flow velocity at point $\mathrm{A}$, the output

speed of ship under measurement, and the actual resultant velocity of ship under measurement.

Based on hypothesis 2, the following equation can be given:

$$
\begin{gathered}
V_{\text {ship }} \times \sin \left(H_{\text {line }}-H\right)-V_{\text {water }} \times \sin \left(\gamma-H_{\text {line }}\right)=0 \\
V_{\text {ship }} \times \cos \left(H_{\text {line }}-H\right)+V_{\text {water }} \times \cos \left(\gamma-H_{\text {line }}\right)-V=0
\end{gathered}
$$

At the same time, it should satisfy the following conditions:

$$
\begin{aligned}
& V_{\text {ship }}+V_{\text {water }}>V \\
& V_{\text {ship }}+V>V_{\text {water }} \\
& V_{\text {ship }}+V>V_{\text {water }}
\end{aligned}
$$

According to hypothesis 1 , the direction and velocity of water flow change little in a short time, which are considered to be constant values in calculating. In actual calculation, $10 \mathrm{~s}$ are used as the time window, $V_{\text {water }}, V_{\text {ship }}$ and $\gamma$ are considered as fixed values within $10 \mathrm{~s}$, then the least-squares solutions of equations from (5) to (9) is obtained.

According to the formula (1), the attitude deviation is related to the actual attitude and the deflection angle. The velocity of water flow and the output speed of ship are two important factors affecting the attitude and deflection

$$
Z_{1} \times\left[1-\frac{\cos \left(\theta_{1}+\delta_{r 1}\right)}{\cos \theta_{1}}\right]-Z_{2} \times\left[1-\frac{\cos \left(\theta_{2}+\delta_{r 2}\right)}{\cos \theta_{2}}\right]=0
$$

In the formula, $Z_{1}$ and $Z_{2}$ represents the water depth values of the sampling points measured at two swaths, $\delta_{r 1}$ and $\delta_{r 2}$ represents the roll deviation of the two swaths at this time, $\theta_{1}$ and $\theta_{2}$ represents the beam angle of the two swaths at the sampling point.

On account of the multi-beam sounding point usually has a certain degree of "thickness", which can reach to several centimeters according to the different type of hardware in use and the condition in measuring area. A method for determining the elevation of a sampling point in the adjacent swath is as follows: the sounding points within $5 \mathrm{~m}$ on the plane distance of the sampling point are screened from two swaths respectively, and then the quadratic plane fitting of these sounding points is carried out to obtain the surface fitting parameters. Finally, the elevation of the sampling point in two adjacent swaths is calculated by using the surface equation determined by fitting parameters.

For a sampling point, because the velocity of water flow and the speed of ship output change slowly, so it can be considered that the roll deviation does not change much in a very short time. Therefore, by selecting multiple points to calculate the height difference in a certain neighborhood of the point to obtain the least-squares solution satisfying equation (10), $\delta_{r 1}$ and $\delta_{r 2}$ can be got.

According to formula 1, attitude deviation is correlated with real-time attitude. At the same time, when installing the error correction at calibration line, the attitude deviation caused by bracket deformation will also affect the correction result. For the above reasons, it is angle, there is a functional relationship between them and attitude deviation. If this function relation is obtained, the velocity information can be got by using the navigation data, then the elevation of sounding point is corrected in accordance with this function relation, which can improve the swath join problem caused by the attitude deviation arisen from the water flow and the speed of ship.

According to formula (2), the equation of height difference value of two swaths caused by roll deviation about a point in the overlapping area of adjacent swaths can be given:

very difficult to derive the expression of attitude deviation from the theoretical point of view, the derived expression will be extremely complex and there will be too many disturbance terms.

There are many variables that affect the attitude deviation, but the main ones are still the direction and size of water flow velocity, the direction and size of ship's output speed. At the same time, since the resultant velocity has been determined, also the vector sum of water flow velocity and the ship's output speed has been determined, so there will be correlation existing in water flow velocity and ship's output speed, there only can be one item in the final expression. Through many experiments, the quadratic function form with $V_{\text {ship }} \times \cos \left(H_{\text {line }}-H\right)$ as independent variable can fit the $\delta_{r}$ in a better way. Among which, $V_{\text {ship }}$ refers to the actual speed of ship, $H_{\text {line }}$ indicates the coordinate azimuth of ship's moving direction, $\mathrm{H}$ represents the coordinate azimuth of ship's direction.

\section{Experiment and analysis}

In May 2018, the multi-beam sounding experiment was carried out in the sea area of Nanji Island, Zhejiang Province, where the water depth is about $22 \mathrm{~m}$, and the sea floor is relatively flat. The measuring ship is 20.6 meters in length and 4 meters in width; the transducer was mounted on the starboard side of measuring ship, it was fixed with a bracket as shown in figure 3 , with a rod length of 2 meters. 


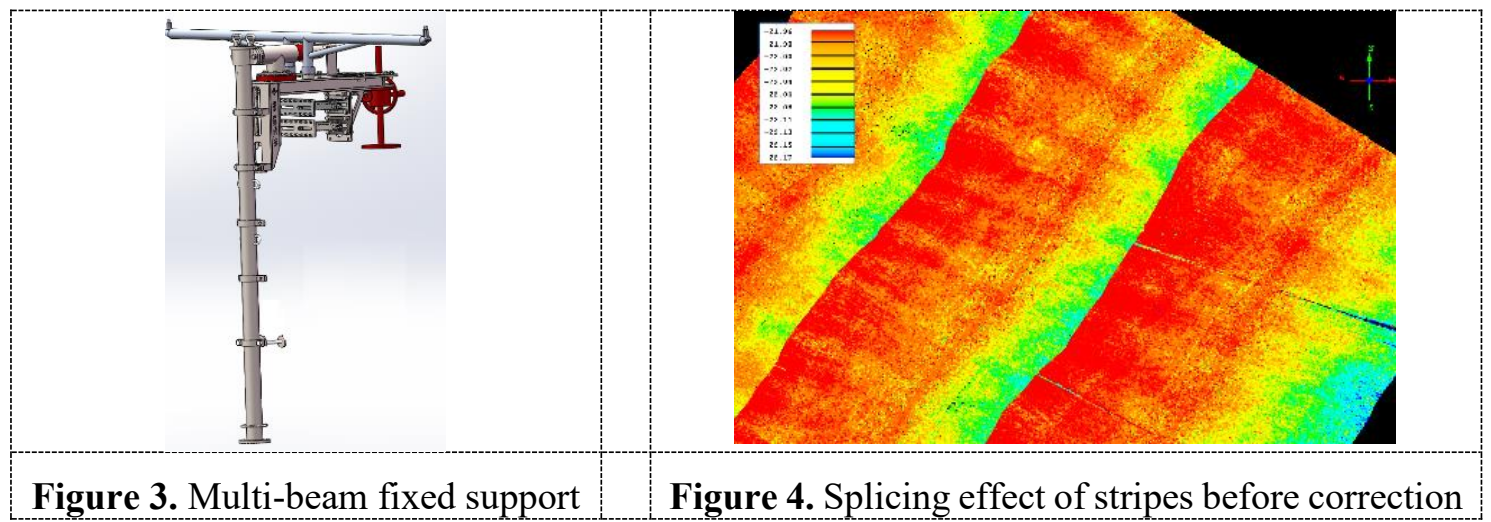

When calculating and solving the measured multibeam data, it is found that the conformity in same swath is good. The overlapping area of adjacent swaths has high difference value and poor effect of swath join. Moreover, the effect of swath join shows different performance in different positions of survey line, which can be joined well

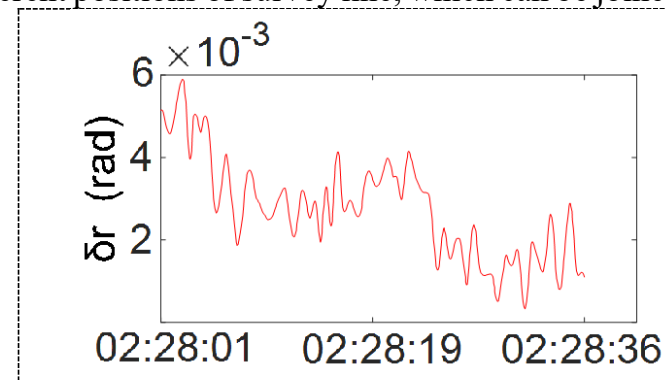

Figure 5. $\delta_{r 1}$ obtained in some areas, but in other areas it presents the phenomenon that one swath is higher than the other, and the swath with high elevation is not fixed. Figure 4 is a typical area of swath join problem, it can be clearly observed that each swath represents obvious incline to the same direction

Through using methods described in section 2 mentioned above to obtain the roll deviation and velocity information of adjacent swaths, figure 5 and figure 6 are partial data (smoothed by using the wavelet filter) after solution. When conducting function fitting on these data, the empirical formula of roll deviation can be obtained by using water flow and ship speed. For all sounding swaths,

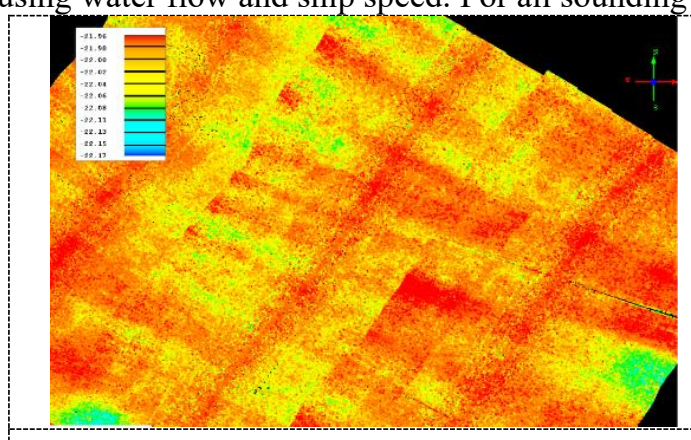

Figure 7. $\delta_{r 1}$ obtained the corresponding curved lines of water flow and ship speed are obtained, then the elevation correction is calculated and corrected by using the empirical formula obtained at all sounding points. As shown in figure 7 , figure 8 , the problem of adjacent swath join is obviously improved. As shown in figure 9, the mean value of swath elevation inconsistency fells from $0.1884 \mathrm{~m}$ to $0.0324 \mathrm{~m}$.

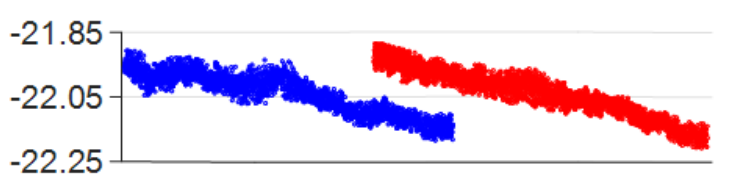

$-21.85$

$-22.05$

$-22.25$

Figure 8. $V_{\text {water }}$, filtered with wavelet 


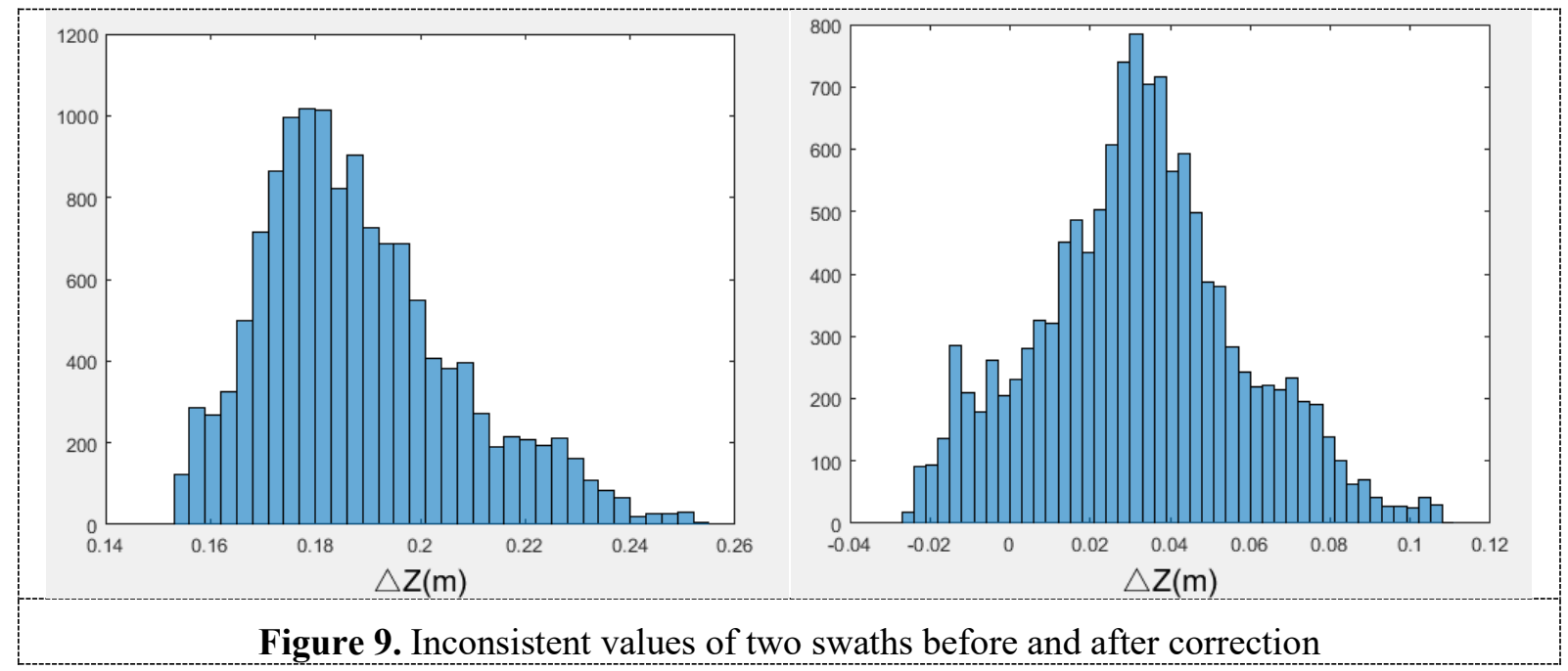

\section{Conclusion}

The multi-beam sounding system can quickly obtain the full-coverage topographic data under the water, but the swath join problem has always been a major obstacle that affects its data application. In this paper, the method of calculating water flow velocity and ship's output speed based on the navigation data is studied, and the method for solving respective roll deviation according to the height inconsistency values of adjacent swath overlapping area is studied, in addition, the roll deviation is represented by water flow velocity and ship's output speed to correct the sounding data. The experimental results show that this method can effectively eliminate the sounding error caused by the attitude deviation due to the change of shafting, which is initially led by the elastic deformation of bracket caused by the impact of water flow.

\section{References}

1. Zhai, G.J., Huang, M.T., Bao, J.Y. (2003) The Requirement and Status of Hydrographic Datum. Hydrographic Surveying and Charting, 04:54-58.

2. Du, M.C. (2009) Means, Accuracy and Utilization of the Capture of Sea-bottom Topographic Data. China Engineering \& Consulting, 09:64-69.

3. Chen, Rui. (2014) Comparison of Installation Methods for Multi-beam Echo Sounder. Ship Science and Technology, 36(S1):79-82.

4. Yang, F.L., Li, J.B., Wu, Z.Y., Zhao, L.H., Ai, Bo. (2009) The Methods of Removing Instantaneous Attitude Errors for Multibeam Bathymetry Data. Acta Geodaetica et Cartographica Sinica, 38(05):450-456.

5. Zhou, Ping. (2017) Research on Error Processing Methods in Mutil-Beam Sounding Swath Joins. East China University of Technology. 